\title{
Epidermolysis Bullosa Nevi: Report of a Case and Review of the Literature
}

\author{
Laura Abdo Nalon de Queiroz Fuscaldi Alice Mota Buçard \\ Carlos Daniel Quiroz Alvarez Carlos Baptista Barcaui
}

Prof. Rubem David Azulay Institute, Rio de Janeiro, Juiz de Fora, Brazil

Key Words

Epidermolysis bullosa · Nevi · Dermoscopy

\begin{abstract}
A 10-year-old female patient, being treated for dystrophic bullous epidermolysis in a Pediatric Hospital, was referred to our Dermoscopy Ambulatory because of a newly observed mole in the submandibular area. Clinically, the lesion presented as an irregular double-colored macule of about $2 \mathrm{~cm}$ in diameter, with irregular borders, suspicious of malignancy. Dermoscopy showed a multicomponent pattern, with multiple colors, ill-defined network, black blotches, streaks, multiple dots, a blue-whitish veil and granularity at the periphery. Although it had a clinical appearance of malignancy, dermoscopy every semester was proposed due to the revision of a recently described entity, named bullous epidermolysis nevi, that we made in these children. The fragile skin of this particular patient was also taken into account, and overtreatment was avoided. Bullous epidermolysis nevi is the term given to large, asymmetrical and often irregularly pigmented melanocytic nevi that occur in former areas of blistering in patients with the dystrophic forms of the disease. Despite its atypical clinical appearance, and sometimes also atypical dermoscopy, malignant transformation has not been reported yet. Similarly to recurrent nevi, where melanocytes proliferate in a previous area of trauma, clinical aspect, dermoscopy, and histopathology may tempt clinicians to diagnose benign moles as melanoma. Here we report one case of this entity, scarcely reported on in literature, and review clinical and dermatoscopical features of epidermolysis bullosa nevi confronting it with recurrent nevi. The usefulness of dermoscopy as a treatment strategy is stressed.
\end{abstract}

\section{Case Report}

A 10-year-old female patient was referred to our Dermoscopy Ambulatory because of a new pigmented lesion noted 6 months before. She was receiving treatment for dystrophic bullous epidermolysis in a Pediatric Hospital. Her mother reported progressive growth and color change of this lesion since its appearance. In clinical examination, the patient presented pseudosyndactily and

Dr. Laura Abdo Nalon de Queiroz Fuscaldi
Rua Padre Café 472/401, Rua Benjamin Colucci $1303^{\circ}$ andar, Centro Juiz de Fora, MG (Brazil)

Tel. +55 323215 8087, E-Mail launalon@gmail.com 
sparse exulcerations in the areas of previous bullae. In the submandibular area, an irregular pigmented mole of about $2 \mathrm{~cm}$ in diameter was observed (fig. 1). Dermoscopy showed a multicomponent pattern, with multiple colors, ill-defined network, black blotches, streaks, multiple dots, a blue-whitish veil and granularity at the periphery (fig. 2). Due to the possibility of a bullous epidermolysis nevi, the authors decided to follow up clinically and with dermoscopy every 6 months. This follow-up was delayed due to infectious complications of the disease, leading to hospitalization of the child.

\section{Discussion}

Epidermolysis bullosa (EB) represents a heterogeneous group of mechano-bullous disorders genetically rooted in an intrinsic defect of the structural components of the basement membrane zone [1].The development of lesions with atypical clinical features in patients with different forms of EB has occasionally been pointed out. The recessive variants of the disease account for the vast majority of the reported cases.

There are a few reports in the literature of moles suspicious of malignancy in patients with bullous epidermolysis, recently classified in a new entity named EB nevi. They commonly arise in areas of previous bullae or erosions, tend to present rapid growth, and usually fulfill the clinical ABCD criteria for atypical or malignant melanocytic proliferations. These nevi are dynamic, beginning as flat pigmented lesions and becoming papillated, loosing pigment or even becoming more pigmented. So far, no evolution to malignancy has been described, but the number of EB nevi studied is not large enough to completely rule out the possibility that they might be melanoma precursor lesions.

Two pathogenic theories about the development of EB nevi have been proposed. According to one of them, the repetitive disruption of the basement membrane leads single melanocytes or local nevus cells to break senescence and undergo proliferation $[2,3]$. According to the other theory, viable melanocytes/nevus cells freely float the cavity of an EB blister [4] and, after settling down, proliferate excessively in the microenvironment of epidermal regeneration in an arbitrary arrangement [5-7]. Cytokines and growth factors, such as interleukin 8, granulocyte-macrophage colonystimulating factor, prostaglandin E2 and leukotriene B4 have been detected in blister fluid [7], which might potentiate melanocytic proliferation. Understanding the pathogenesis may help disclose their benign nature, in spite of false-positive scores with application of dermoscopic algorithms.

Interestingly, EB nevi show analogy to recurrent nevi after incomplete surgical excision or trauma of a nevus. Such traumatized moles also frequently mimic melanoma, clinically, dermoscopically and histologically. While dermoscopy and, more recently, reflectance confocal microscopy (RCM) have proven to be valuable tools in the diagnosis of most pigmented lesions, little is currently known about their clinical value in the differential diagnosis of recurrent melanocytic proliferations. Longo et al. [8] investigated dermoscopic and RCM features of seven histopathologically diagnosed cases of recurrent melanocytic proliferations. Repigmentation occurring within the scar through dermoscopy was a clue suggestive for nevi, while a lateral spreading of the pigmentation, extending from the border of the scar towards the surrounding normal skin was observed in all cases of melanoma. Although scar pigmentation is a frequent phenomenon, there are few papers on this subject [9]. Yoshida et al. [10] reported the 
case of a 47-year-old patient with a mole on her left arm since childhood, where a darker pigmentation had been observed. Through dermoscopy a starburst pattern was seen, and histopathology diagnosed a melanocytic nevus with partial recurrence after minor trauma. Similarly to what happens in EB nevi, it was speculated that stimulation of melanin production might have been induced in the wound healing process.

Lanschuetzer et al. [11] used dermoscopy to test usefulness of this technique in distinguishing EB nevi from melanoma. Twenty-three nevi of 11 patients were analyzed with a dermoscope. Twenty of the 23 lesions were classified as having a multicomponent pattern or showed a 3-structure type pattern, both highly suggestive of malignant melanoma. More than half of the lesions achieved a high score for the $\mathrm{ABCD}$ and 7-point checklist. Nevertheless, strong morphologic indicators for invasive cutaneous melanoma, or a blue-whitish veil, which represents a melanoma-induced acanthotic epidermis, were not observed. Although it was often possible to estimate the benign nature of the moles, unequivocal discrimination from melanoma could not be made, and regular follow-up was proposed.

Schiffner et al. [12] proposed a progression model of lentigo maligna located on the face. According to their findings, slate-gray dots are an early feature of lentigo maligna, which can increase to globules. Short streaks can be detected, and can progress to very specific rhomboidal structures. Melanoma cells within the follicles can invade the adjacent dermis, resulting in bizarre globules at the periphery of the follicle. During further tumor progression, rhomboidal structures become broader and hair follicles are obliterated.

Taking into account all the aspects of EB nevi described above, it can be considered an exception among melanocytic lesions. If not aware of this particular nevi, clinicians might be tempted to establish a more aggressive treatment, when clinical and dermoscopic semestral review would be a secure and more convenient therapeutic strategy for these fragile skin patients. Here dermoscopy presents as an essential technique to prevent overtreatment. 


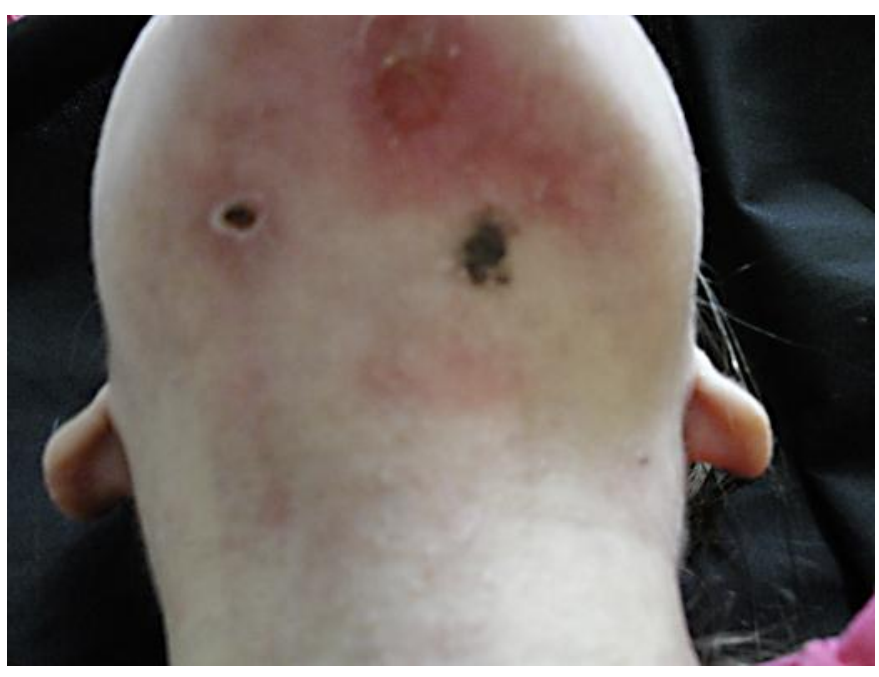

Fig. 1. Clinical aspect of the lesion.

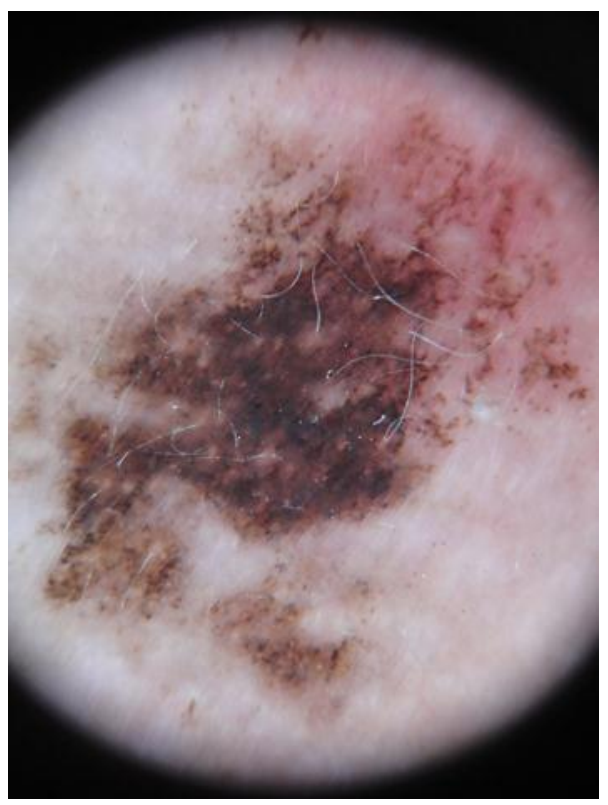

Fig. 2. Dermoscopic aspect of the mole. 


\section{References}

$>1$ Botella-Estrada R, Nagore E, Sopena J, Cremades A, Alfaro A, Sanmartín O, Requena C, Serra-Guillén C, Guillén C: Clinical, dermoscopy and histological correlation study of melanotic pigmentations in excision scars of melanocytic tumours. Br J Dermatol 2006;154:478-484.

2 Gllardo F, Toll A, Malvehy J, Mascaró-Galy JM, Lloreta J, Barranco C, Pujol R: Large atypical melanocytic nevi in recessive dystrophic epidermolysis bullosa: clinicopathological, ultrastructural and dermoscopic study. Pediatr Dermatol 2005;22:338-343.

3 Lanschuetzer CM, Laimer M, Nischler E, Hintner H: Epidermolysis bullosa nevi. Dermatol Clin 2010;28:179-183.

-4 Hoss DM, McNutt NS, Carter DM, et al: Atypical melanocytic lesions in epidermolysis bullosa. J Cutan Pathol 1994;21:164-169.

5 Soltani K, Pepper MC, Simjee S, Apatoff BR: Large acquired nevocytic nevi induced by the Koebner phenomenon. J Cutan Pathol 1984;11:296-299.

-6 Bauer JW, Schaeppi H, Kaserer C, Hantich B, Hintner H: Large melanocytic nevi in hereditary epidermolysis bullosa. J Am Acad Dermatol 2001;44:577-584.

-7 Grubauer G, Hintner H, Klein G, Fritsch P: Acquired surface giant nevus cell nevi in generalized, atrophic, benign epidermolysis bullosa (in German). Hautarzt 1989;40:523-526.

$>8$ Longo C, Moscarella E, Pepe P, Cesinaro AM, Casari A, Manfredini M, Stanganelli I, Gardini S, Cota C, Argenziano G, Pellacani G, Zalaudek I: Confocal microscopy of recurrent naevi and recurrent melanomas: a retrospective morphological study. Br J Dermatol 2011;165:61-68.

-9 Lanschuetzer CM, Emberger M, Hametner R, et al: Pathogenic mechanisms in epidermolysis bullosa naevi. Acta Derm Venereol 2003;83:332-337.

10 Yoshida Y, Yamada N, Adachi K, et al: Traumatized recurrent melanocytic naevus with typical starburst pattern on dermoscopy. Acta Derm Venereol 2008;88:408-409.

11 Lanschuetzer CM, Emberger M, Laimer M, Diem A, Bauer JW, Soyer HP, Hintner H: Epidermolysis bullosa naevi reveal a distinctive dermoscopic pattern. Br J Dermatol 2005;153:97-102.

$\$ 12$ Schiffner R, Schiffner-Rhoe J, Vogt T, Landthaler M, Wlotzke U, Cognetta AB, Stolz W: Improvement of early recognition of lentigo maligna using dermatoscopy. J Am Acad Dermatol 2000;42:25-32. 